\title{
Propagation characteristics of arrayed transient electromagnetic pulses
}

\author{
CUI HaiJuan*, YANG HongChun, RUAN ChengLi \& WU MingHe \\ School of Physical Electronics, University of Electronic Science and Technology of China, Chengdu 610054, China
}

Received October 25, 2010; accepted March 4, 2011; published online May 12, 2011

\begin{abstract}
Starting from the uniform disk current model, an analytical solution for the electromagnetic pulse axial energy radiation is derived and a physical meaning then given to the axial energy propagation characteristics of a unit antenna. From this solution, a point-source approximation model in the time domain is introduced. Parameters such as the energy propagation characteristics and beamwidth of the electromagnetic pulse beam radiating from arrayed antenna are analyzed theoretically. An arrayed transient electromagnetic pulse generator is developed incorporating a $23 \times 16$ ultra-wideband tapered slot antenna integrated with a Blumlein formation line and photoconductive semiconductor switch. Experiments performed over a $2.5 \mathrm{~km}$ range confirm that the arrayed transient electromagnetic pulse can propagate in free space with the specified waveform. Moreover, the field components can be superposed in phase, and the radiation beam can be focused into a single pulse. Results from calculations are in agreement with experimental results within the measurement uncertainties.
\end{abstract}

arrayed transient electromagnetic pulse, point-source approximation model, propagation characteristics

Citation: Cui H J, Yang H C, Ruan C L, et al. Propagation characteristics of arrayed transient electromagnetic pulses. Chinese Sci Bull, 2011, 56: 1957-1964, doi: $10.1007 / \mathrm{s} 11434-011-4457-4$

The ongoing developments of telecommunication and the ever-increasing demand on radio frequency spectrum resources have led to the development of ultra-wide bandwidth electromagnetic technologies. This has once again forced the development of electromagnetic pulses in the time domain $[1,2]$. For this, the key technologies and issues are: (1) An electromagnetic pulse generator with high peak power, low trigger jitter, fast response and long life time. Because of the difficulties in limiting trigger jitter to picosecond-levels, superimposing the pulses power from an arrayed generator is hard; (2) Microwave devices with ultra-wide bandwidth. To use electromagnetic pulse resources in the time domain, electromagnetic pulses should remain undistorted in intensity, propagation, and reception. Ultra-wide bandwidth circuitry and devices must work over low to high frequency ranges; and (3) Basic theoretical research on the characteristics of the radiation and propaga-

*Corresponding author (email: cuihaijuan@126.com) tion of the transient electromagnetic pulses. On the one hand, the theory of antenna in the time domain presumes that the antenna theory in the frequency domain poorly describes the characteristics of antenna in the time domain; introducing new parameters and new theories are necessary. On the other hand, electromagnetic theory presumes that transient electromagnetic pulses are characterized by slow energy decay, spatial superposition of field components and beam focusing. These characteristics are distinct from those of radiation and propagation of continuous sine waves. Over the past thirty years, significant work has been performed in these key technologies and much progress have been made in important theoretical and technological developments such as photoconductive semiconductor switches (PCSS) [3-9], antenna theory and technology in the time domain $[10,11]$, and some applied basic theories such as the propagation theory of arrayed transient electromagnetic pulses and some key technologies [12-15]. The results of all this research have been used for ground penetrating radar [16], 
high power microwaves [9,17], THz technology $[7,18]$, and biomedicine [19,20].

Based on the analytical current-model solution for a transient electromagnetic pulse radiating from a uniform disk, the physical nature of the axial energy propagation characteristics radiating from a unit antenna is given. A point-source approximation model in the time domain is introduced and the electromagnetic pulse beam width and energy propagation characteristics of the arrayed antenna are analyzed theoretically using this model. As a result of breakthroughs in key technologies, such as the lifetime of high-power PCSS [21-23], we developed a $23 \times 16$ arrayed electromagnetic pulse generator that employs laser excitation synchronization techniques via focused beam splitter, optoelectrical synchronizers, opposed-surface ultra-wideband antennas integrated with a Blumlein formation line and a miniature pulsed high-voltage power supply. This pulse generator was used in carrying out experimental measurements over a distance of $2.5 \mathrm{~km}$. We confirmed that the radiated transient electromagnetic waves can be transmitted in free space with characteristics including slow energy decay, in-phase superposition, and beam focusing.

\section{Analysis of the propagation characteristics of transient electromagnetic pulse beams}

\subsection{Analytical solution of electromagnetic pulse radi- ating from a unit antenna}

Consider a bow-tie antenna fed with a transient electric pulse. In $[22,24]$ the electric field in the polarization direction (shown in Figure 1(a)) was shown to be approximately equivalent to the radiation field from a time-varying current $j(t)$ distributed uniformly along one direction. Similarly, the electromagnetic radiation from a parabolic antenna (shown in Figure 1(b)) fed with a bow-tie antenna located at the parabolic focus is approximately equivalent to radiation from a time-varying current $j(t)$ distributed uniformly along one diameter of a disk with the same aperture. According to this abstract model, radiation from antennas can be simply modeled as electromagnetic radiation from a time-varying current $j(t)$ distributed uniformly along the same direction. Under this assumption, parabolic antenna radiation is seen as radiation from a time-varying current distributed uniformly along the disk. The axial energy can be calculated according to Maxwell's equations and written as

$$
\begin{aligned}
G= & \frac{\eta}{4} \int_{-\infty}^{+\infty}\left[g_{1} g_{2} f^{2}\left(t-\frac{R}{c}\right)+f^{2}\left(t-\frac{z}{c}\right)\right] \mathrm{d} t \\
& -\frac{\eta}{4} \int_{-\infty}^{+\infty}\left(g_{1}+g_{2}\right) f\left(t-\frac{R}{c}\right) f\left(t-\frac{z}{c}\right) \mathrm{d} t,
\end{aligned}
$$

where

$$
\eta=\frac{1}{\varepsilon c}, g_{1}=\frac{z}{R}, g_{2}=\frac{1}{2}\left(1+\frac{z^{2}}{R^{2}}\right)
$$
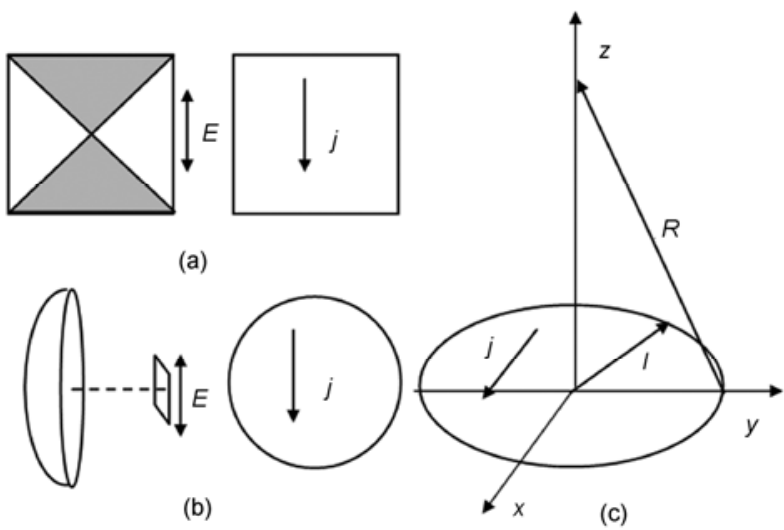

(b)

(c)

Figure 1 Uniform disk current model for analyzing unit antenna. (a) Physical model of a bow-tie antenna; (b) physical model of parabolic antenna; (c) circular radiating antenna model.

$$
R=\sqrt{a^{2}+z^{2}}, \xi=\frac{R-z}{c}
$$

the antenna aperture is $2 l, f(t)$ is the electrical pulse waveform radiated from the antenna, and $c$ is the velocity of light in a vacuum. From eq.(1), we can conclude that the radiation from a radiating circular antenna is composed of two transient electromagnetic pulses, one radiating from the circle center and the other from a random point on the circumference. Within time interval $\Delta t$, the energy of a unit cross-sectional area flowing through axial point $z$ is equal to the sum of Poynting-vector integrations with respect to time of the two transient pulses, minus their interference. Thus, the axial energy of the antenna radiates in three propagation regimes depending on propagation distance: energy invariance, slow decay, and rapid decay [24]. When $z>>l$, we can conclude from eq. (1) that

$$
G \approx \frac{\eta}{4} \int_{-\infty}^{+\infty} f^{2}(t) \mathrm{d} t \cdot \frac{l^{2}}{z^{2}} \propto \frac{1}{z^{2}}
$$

which is just the decay law for energy radiating from a point source antenna in the far-field region. Thus, in this region, we can treat the unit antenna as a radiating point-source. Furthermore, the focus wave mode theory [12] has shown that the transient electromagnetic pulse is propagating as a three-dimensional directed pulse in free space at the velocity of light. In this manner, the theoretical calculation of the electromagnetic pulse radiation from an antenna array can be described as the spatial superposition of radiation from each point-source antenna.

For an antenna element, the far-field region is defined by propagation distances $z_{y}>l^{2} / \mathrm{c} T$; for pulses of width $T=1.8 \mathrm{~ns}$, $z_{y}>0.0417 \mathrm{~m}$ given aperture $2 l=0.3 \mathrm{~m}$, so even at this rather close distance, we can treat the unit antenna as a radiating point source.

\subsection{Point-source approximation model}

From the physical interpretation of eq. (1) and focus wave 
mode theory, the point-source approximation model in time domain can be summarized as (1) the transient electromagnetic pulse is propagating out as a three-dimensional pulse in free space; (2) the field component amplitude of the transient electromagnetic pulse is inversely proportional to its propagation distance; (3) at a specific measurement point, the radiation from a $m \times n$ antenna array can be regarded as the superposition of field components radiating from $m \times n$ point-source antennas; (4) within a time $t$, the electromagnetic pulse energy flowing through the measurement point is proportional to the temporal integration of the square of the field component of the composite pulse, which is a superposition at this point of pulses from all point sources; and (5) the couplings among the point- source antenna units are ignored.

According to the point-source approximation model, the time-domain waveform of the electromagnetic pulse from the antenna array at the measurement point is

$$
E\left(r_{i j}, t\right)=E_{0} r_{0} \sum_{i, j} \frac{1}{r_{i j}} f\left(t-\frac{r_{i j}}{c}, \Omega\right),
$$

where $\left(x_{i}, y_{j}, 0\right)$ is the coordinate of the element located in the $i$ th row and $j$ th column of the array, and the measurement point coordinate is $(x, y, z)$. In addition, $E_{0}$ is the electric field radiated from unit antenna at the furthermost axial point $r_{0}\left(0,0, z_{0}\right)$ within the energy invariance regime, while $f\left(t-r_{i j} / c, \Omega\right)$ is the advanced waveform function at angular angle $\Omega$ given time $t$ and distance $r_{i j}$ from the array center defined by

$$
r_{i j}=\sqrt{\left(x-x_{i}\right)^{2}+\left(y-y_{j}\right)^{2}+z^{2}} .
$$

From eq. (4), the ratio of the pulse energy flowing through the measurement point $z$ to that at the reference point $r_{0}$ with duration $T^{\prime}$ can be written as

$$
\frac{G\left(r_{i j}\right)}{G\left(r_{0}\right)}=\int_{0}^{T^{\prime}}\left[\sum_{i, j} \frac{r_{0}}{r_{i j}} f\left(t-\frac{r_{i j}}{c}, \Omega\right)\right]^{2} \mathrm{~d} t .
$$

\subsection{Point-source approximation model calculations of beam parameters of the electromagnetic pulse}

(1) Weak coupling condition between array elements in the time domain Assume the full-width at half-maximum (FWHM) of the electromagnetic pulse from unit antenna is $T$ and the spacing between antenna units is $d$. According to the Rayleigh criteria on the minimum distinguishable distance between two transient electromagnetic pulses in optics, the minimum spacing between the elements in the array should satisfy

$$
d \geqslant \frac{c T}{2}
$$

Under this weak coupling condition, the minimum spacing between the elements can be calculated using eq. (7) given $T$. If the minimal coupling condition is satisfied, the density of the array can be increased if the elements are arranged in an alternating pattern.

(2) Energy propagation characteristics of transient electromagnetic pulse beams A "three-regime" propagation is the universal rule followed by transient electromagnetic pulse energy propagation [24], and also by pulse from an antenna array. Assume the aperture of the antenna array is $2 l$. If the electromagnetic pulse radiating from the array is propagating in the near-field region, the propagation distance is small; if the maximum time difference $\Delta t$ among pulses successively arrive at the measurement point is larger than $T$, that is

$$
\sqrt{z^{2}+a^{2}}-z \geqslant c T
$$

pulses radiating from each of the elements will either pass the measurement point $z$ independently or only partially be in-phase and superimpose at $z$. The beam width of the pulse radiating from the array will then be wide at $z$, the energy will remain constant or approximately so over a certain range in the axial direction. From eq. (8) we derive the condition

$$
z \leqslant \frac{a^{2}-c^{2} T^{2}}{2 c T}
$$

that determines the energy-invariance distance range.

If the propagation distance is increased to the nearintermediate-field region, the maximum time difference $\Delta t$ among pulses successively arrive at $z$ decreases gradually. When the time difference is smaller than $T$ but still larger than the rise time $\tau$ of the radiated pulse of unit antenna, we have constraint

$$
c \tau \leqslant \sqrt{z^{2}+a^{2}}-z \leqslant c T .
$$

According to the Raleigh criterion, the two transient electromagnetic pulses just lie between being distinguishable and indistinguishable under this constraint. Increasing $z$ decreases the time difference $\Delta t$, so that more elements will contribute to the composite electromagnetic pulse, with a pulse width that is rapidly reduced. However, the electric field from each of the elements is inversely proportional to distance $z$ according to the point-source approximation model. The two effects result in a superimposed pulse that is characterized by a slow energy decay, the decay falling off slower than $z^{-2}$ but faster than $z^{-1}$, and hence

$$
\frac{a^{2}-c^{2} T^{2}}{2 c T} \leqslant z \leqslant \frac{a^{2}-c^{2} \tau^{2}}{2 c \tau},
$$

corresponding to the slow energy decay distance criteria. In practice, the physical nature of this slow energy decay stems from the in-phase superposition of the pulses from each of the elements in conjunction with energy decay of $z^{-2}$. 
Further, increasing the propagation distance into the far-intermediate-field region, the time difference $\Delta t$ becomes smaller than the rise time $\tau$, that is

$$
\sqrt{z^{2}+l^{2}}-z \leqslant c \tau \text {. }
$$

The electromagnetic pulses from each of the elements can be regarded as "fully" superimposed forming a single transient electromagnetic pulse, the superposition remaining almost constant with continued increase in propagation distance. Thus, in this range, the pulse width from this array will remain constant and correspond to the electromagnetic pulse width of the unit antenna. In addition, the energy of this superimposed electromagnetic pulse is inversely proportional to the square of the distance, that is

$$
z \geqslant \frac{a^{2}-c^{2} \tau^{2}}{2 c \tau},
$$

which provides the range condition for the rapid energy decay distance.

According to the point-source approximation model and the Rayleigh criteria, distance criteria for the three propagation regimes, energy invariance, slow decay, and rapid decay, are given in eqs. (9), (11), and (13), respectively. These are in good agreement with [22,24] where the results were analytically derived from Maxwell's theory of electromagnetism. However, the above analysis indicates that the characteristics of the three regimes are the result of pulse superposition from each of the elements, which is in contrast to the suggestive "intrinsic characteristics" of continuous electromagnetic waves. Both the continuous electromagnetic waves and the transient electromagnetic pulses must obey the classical Maxwell theory. It should be noted that the distance criteria for energy invariance, slow decay and rapid decay are theoretical results obtained according to their respective physical assumptions. In fact, these are continuous criteria and there is no clear separation between them.

(3) Radiation patterns of transient electromagnetic pulse beams in the time domain. For an antenna waveform in the time domain, the frequency spectrum is rather rich and there are major differences in patterns and gains for different frequencies. These parameters are inconvenient to use in the frequency domain to describe the spatial energy distribution of the arrayed transient electromagnetic pulses, but also, the energy density is difficult to describe at the target position. For this reason, we introduce the radiation pattern in the time domain based on the point-source approximation model for the arrayed antenna. A half-power beamwidth is defined as the angular separation $\theta_{1}$ of the points where the superimposed field density drops to $70.7 \%$ of the maximum value at the central axis for the same distance $z$. The pattern is defined as either the peak field component of the electromagnetic pulse or peak power as a function of $\theta$, where half of $\theta$ is the angle of the central axis of the superimposed pulse deviation from the central axis of the array for the same distance.

According to the point-source approximation model and the Rayleigh criteria, the half-power beam width of the electromagnetic pulse beams should satisfy:

$$
\theta_{1} \leqslant \arcsin \frac{c T}{0.707 l}
$$

Clearly from eq. (14), the beam width is reduced if the aperture of the array is increased under a specific arrangement of elements. The focusing property of the superimposed transient electromagnetic pulse beam from array can, therefore, be easily understood.

\section{The arrayed transient electromagnetic pulse generator}

Figure 2 shows the configuration of the arrayed transient electromagnetic pulse generator. This works as follows: the two TTL signals from an optoelectrical synchronization device are used to control the trigger time of the laser and the high-voltage pulsed power supply. By controlling the time delay between the two level signals and as the pulsed high-voltage bias on the PCSS reaches its peak amplitude, the PCSSs are excited simultaneously, to within a synchronization accuracy of ps, by the $n$ laser beams from the 1-to- $n$ focused beam splitter. The high-power and high-stability transient electrical pulses are generated from the PCSSs. These high-power transient electrical pulses after reshaping by the Blumlein formation lines were fed to an ultra-wide bandwidth antenna array to form the high-power ultra-wide bandwidth electromagnetic pulse beam. The propagation characteristics of the electromagnetic pulses were measured using an ultra-wide bandwidth receiving antenna.

A miniature high-voltage pulsed power supply was developed by employing back-scanning charging transformation technology [25]. The precision in the delay time between the two TTL signals from the optoelectrical synchronization device is $1 \mu \mathrm{s}$, the maximum time delay being $1 \mathrm{~ms}$ [26]. The joins of the focusing beam splitter were connected

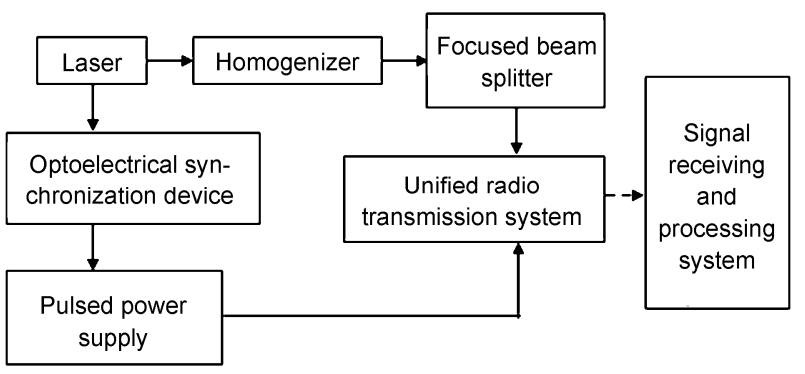

Figure 2 Configuration for the arrayed transient electromagnetic pulse generator. 
in a hexagonal pattern, the laser energy is made uniform by a homogenizer before entering the beam splitter so that the energies within the beam splitter are uniformly distributed. The error in the time synchronization of the $n$ laser pulses is less than 3 ps [27]. Figure 3(a) shows a $3 \mathrm{~mm}$ opposed-contact GaAs PCSS following a Rogowski-profile electrode; the improved Blumlein structure integrated with a PCSS is shown in Figure 3(b), the thickness of the single layer FR4 substrate being $1.5 \mathrm{~mm}$, and the relative dielectric constant $\varepsilon_{r}=4.2$. The Blumlein line acts as a energy storage (energy storage capacitor), wave modulator (controlling the width of the electric pulses), and double circuit voltage transmission line. Figure 4 shows the configuration of the unified antenna based on PCSS integrated with Blumlein formation line and ultra-wideband tapered slot antenna. There are two layers of media for the radiation antenna, the two wings of the antenna are in an opposed arrangement on the front and back faces separately, as well as a part of the Blumlein transmission line. The ground plane of the Blumlein line is inserted between the two FR4 layers with a

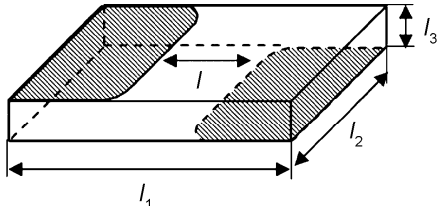

(a)

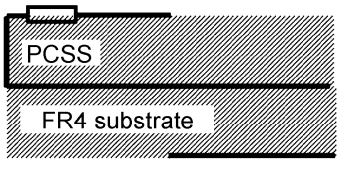

(b)
Figure 3 Geometry of a PCSS based on the Blumlein formation line. (a) GaAs PCSS following a Rogowski profile electrodes; (b) PCSS integrated with Blumlein.

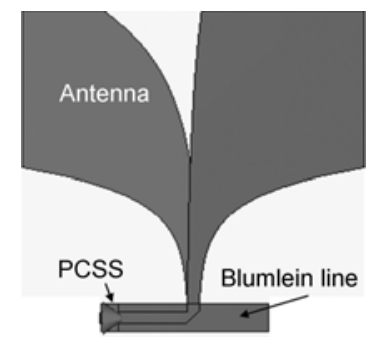

Figure 4 Unified geometry of the asymmetrical antenna.

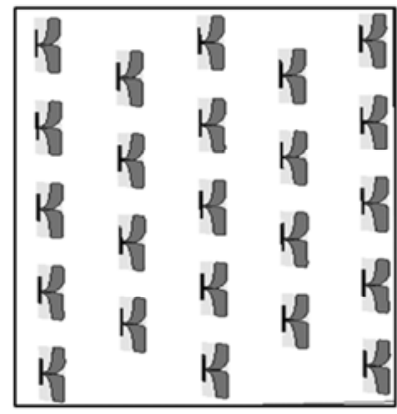

(a)

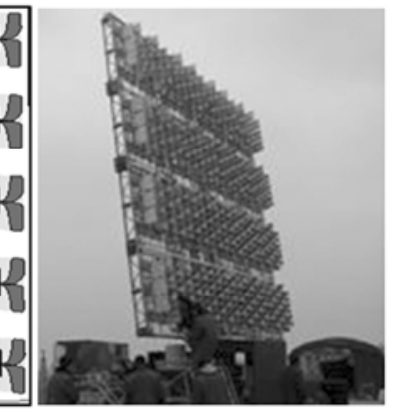

(b)
Figure 5 (a) Configuration of a subarray; (b) system consists of $4 \times 4$ subarrays. thickness of 3 times the width of the transmission line. Both impedances of the tapered slot antenna (input) and the Blumlein formation line (output) are $40 \Omega$. Taking the $6 \mathrm{~dB}$ as a reference, the bandwidth of the radio emission of this unified antenna system is $240 \mathrm{MHz}-2 \mathrm{GHz}$. The gain at the central frequency is $4 \mathrm{dBi}$.

Figure 5(a) shows the structure of a subarray of the radiating system. Each subarray includes 23 unified radiating antennas in a alternating arrangement. The dimension of the subarray is $1.8 \mathrm{~m} \times 1.8 \mathrm{~m}$. The system shown in Figure 5(b) consists of $4 \times 4$ subarrays.

\section{The experimental measurements and analysis}

\subsection{Tests and analysis on the waveform parameters of the transient electromagnetic pulses}

According to the point-source approximation model, the path differences among electromagnetic pulses radiated from the array elements gradually decrease as the measured axial distance $z$ increases. These electromagnetic pulses are superimposed into an indistinguishable composite pulse. Therefore, the measured composite electromagnetic pulse width at the axial point is reduced by degrees and eventually tends to the width of a pulse radiating from a single element as measured from the experiment.

Experimental results (shown in Figure 6) from the system of Figure 5(b) show that, in the vicinity of axial distance $z=50 \mathrm{~m}$, the received pulse width has decreased rapidly. As the axial distance increases further, the width of the pulse is reduced and approaches the pulse width for a unit antenna of $1.8 \mathrm{~ns}$ (a typical received pulse for $z>100 \mathrm{~m}$ is shown in Figure 6(a)). Figure 6(b) plots the calculated pulse waveform radiating from the experiment system at different axial distances $z$ using the point-source approximation model. Figure 6(c) presents the experimental and computational pulse width from the 368 antenna array system depicted in Figure 5(b). In the far-field region, $z>100 \mathrm{~m}$, the calculated results from our model are in agreement with experimental measurements, confirming the validity of the point-source approximation model in analyzing the width of the electromagnetic pulse from this antenna array. The two results deviate over axial distances $30-50 \mathrm{~m}$, possibly because the radiated pulse waveform in time domain will be different for antennas in different direction of $\mathrm{E}$ - or $\mathrm{H}$ planes (which is not considered in our theoretical calculations); when axial distances are not so great, the trigger jitter and field amplitude jitter of each of the elements will have significant impact on the array synchronization in time. Coupling between the radiating array and the receiving antenna was not taken into account; and the geometry but not effective aperture of the antenna array was used in theoretical calculations. 

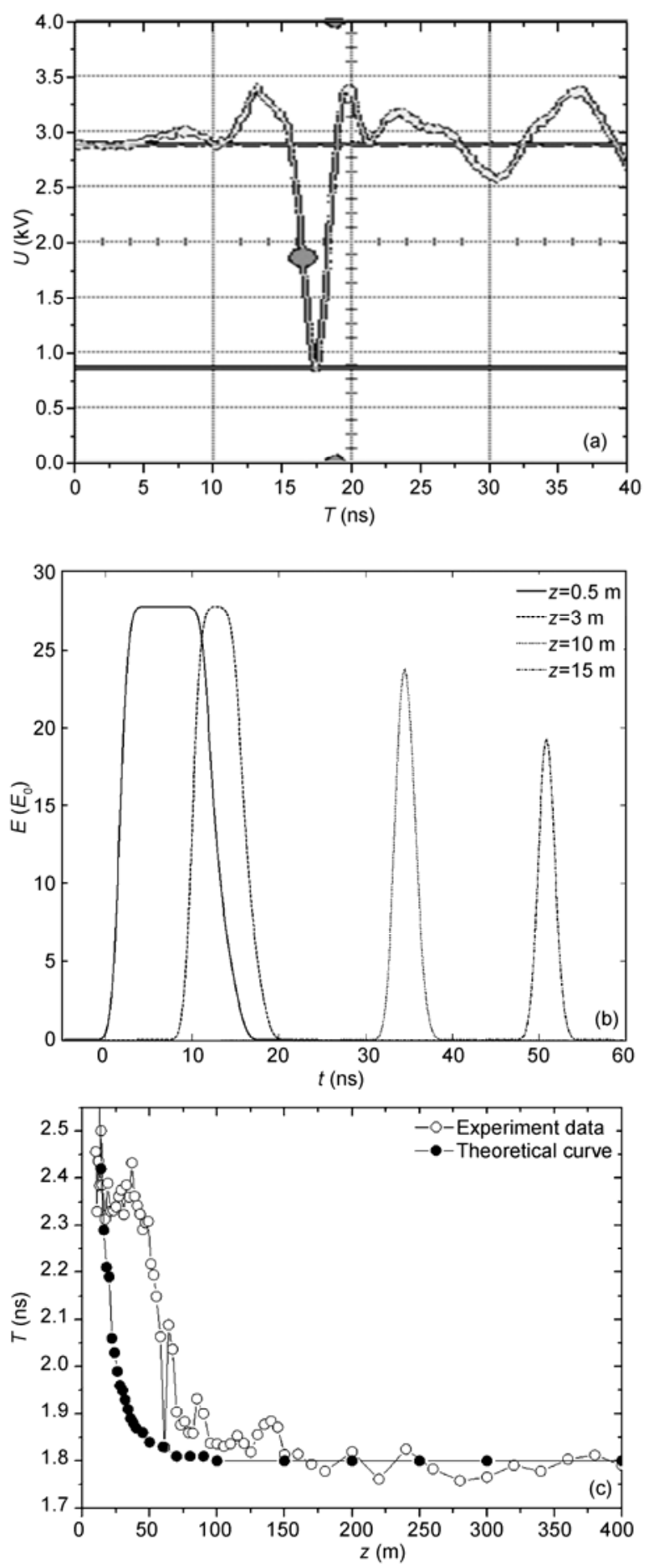

Figure 6 (a) The received electromagnetic pulse waveform at $z=100 \mathrm{~m}$; (b) the calculated pulse waveform at different axial distance; (c) measured and calculated pulse width from a 368 antenna array dependence on the axial distance $z$.

\subsection{Measurements and analysis on the energy propagation characteristics}

The axial energy as a function of axial distance from the $4 \times 4$ subarray system both from experimental tests and theoretical calculations are shown in Figure 7. This features (1) the "three-stage" regimes of the superimposed electromagnetic pulse with axial distance: invariance, slow decay and rapid decay regimes; and (2) the good agreement, within the measurement uncertainties, of the experimentally-measured distance ranges for invariance, slow decay and rapid decay regimes of the superimposed electromagnetic pulses, with calculated results using eqs. (9), (11), and (13) obtained from the point-source approximation model (The electromagnetic pulse is approximated by a Gaussian function with pulse width $T=1.8 \mathrm{~ns}$, and antenna array geometry aperture $2 l=7.8 \mathrm{~m}$.). In the rapid decay regime, the theoretical results show that the energy is inversely proportional to the squared distance whilst the experimental results indicate that the energy decay rate is $z^{-3}$, or even faster than $z^{-3}$. This deviation can be explained by ground reflections in the far field (shown in Figure 8).

Given the electromagnetic pulse waveform $E_{0}(z)$ radiated from a unit antenna, let $E_{1}(z)$ denote the reflected electromagnetic pulse waveform emanating from some point in the ground. Taking the half-wave loss of the ground reflection into consideration, the received time-domain waveform in the receiving antenna is

$$
\begin{array}{r}
E(z)=E_{0}(z)-E_{1}(z)=\frac{E_{0}}{z} \exp \left[-\alpha\left(t-\frac{z}{c}\right)^{2}\right] \\
-\frac{E_{0}}{\left(r_{1}+r_{2}\right)} \exp \left[-\alpha\left(t-\frac{r_{1}+r_{2}}{c}\right)^{2}\right],
\end{array}
$$

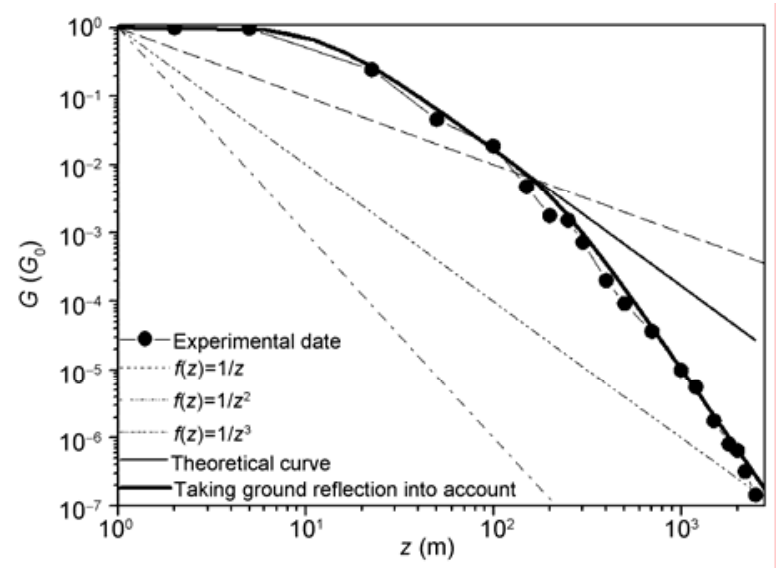

Figure 7 Array energy dependence on the axial distance.

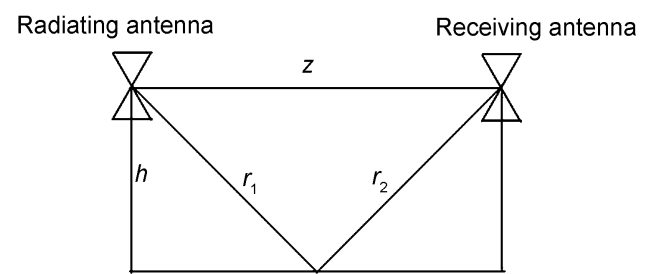

Figure 8 The ground reflection influence. 
where $h$ is the height of the antenna above ground and

$$
r_{1}=r_{2}=\sqrt{\left(\frac{z}{2}\right)^{2}+h^{2}} .
$$

Eq. (15) reaches its extreme point when $t=t_{0}$, where $t_{0}$ should satisfy

$$
\frac{2 r_{1}}{z} \frac{t_{0} c-z}{t_{0} c-2 r_{1}}=\mathrm{e}^{-\frac{4 \alpha h^{2}}{c^{2}}} \mathrm{e}^{\frac{4 \alpha h^{2} t_{0}}{z c}} .
$$

When $z>>l$ and $z-2 r_{1} \rightarrow 0$, eq. (15) can be simplified as

$$
\frac{E(z)}{E_{0}}=\frac{1}{z}\left(1-\mathrm{e}^{-\frac{4 \alpha h^{2}}{c^{2}}} \mathrm{e}^{\frac{4 \alpha h^{2} t}{z c}}\right) .
$$

To substitute eq. (16) into eq. (17) when $t=t_{0}$, we can get

$$
\frac{E(z)}{E_{0}}=\frac{1}{z}\left(1-\frac{2 r_{1}}{z} \frac{t_{0} c-z}{t_{0} c-2 r_{1}}\right) .
$$

If set $\left(t_{0} c-z\right) /\left(t_{0} c-2 r_{1}\right) \approx 1$, then

$$
\frac{G(z)}{G(0)} \propto\left[\frac{E(z)}{E_{0}}\right]^{2} \approx \frac{4 h^{4}}{z^{6}} .
$$

When there is ground reflection, we can conclude from eqs. (18) and (19) that the axial energy decay rate of arrayed transient electromagnetic pulse with $z$ is faster than $z^{-2}$, even faster to $z^{-6}$, which is just the reason why the measured energy decay rate in Figure 7 is $z^{-3}$ and the decay rate measured in literatures is $z^{-4}$. Because of $E(z) / E_{0}$ is rather sensitivity to $t_{0}$ in eq. (18), Figure 7 also gives the calculation results according to the experiment parameters and eq. (15). The calculation results agree well with the experiment results, it confirms that the energy decay rate of transient electromagnetic pulse with $z$ is $z^{-3}$, or even faster than $z^{-3}$, which can be explained by the ground reflection.

\subsection{Tests and analysis on the beam focusing character- istics of the transient electromagnetic pulses}

Detailed calculations and analysis on the focusing characteristics of the transient electromagnetic pulses have been reported in [21]. Experimental tests on these characteristics are important as the angular resolution of radars is dependent of the width of the transient electromagnetic pulses.

The radiation patterns of the antenna array (shown in Figure 5(b)) in the H-plane have been measured experimentally. During measurements, the receiving antenna is at the same height above ground as the array center. The data were collected at the receiver end and at regular intervals along a straight line that is vertical to the central axis and parallel to the plane of the antenna array, as well as to the right hand side of the array. Figure 9 shows the results of experimental measurements and computational results using

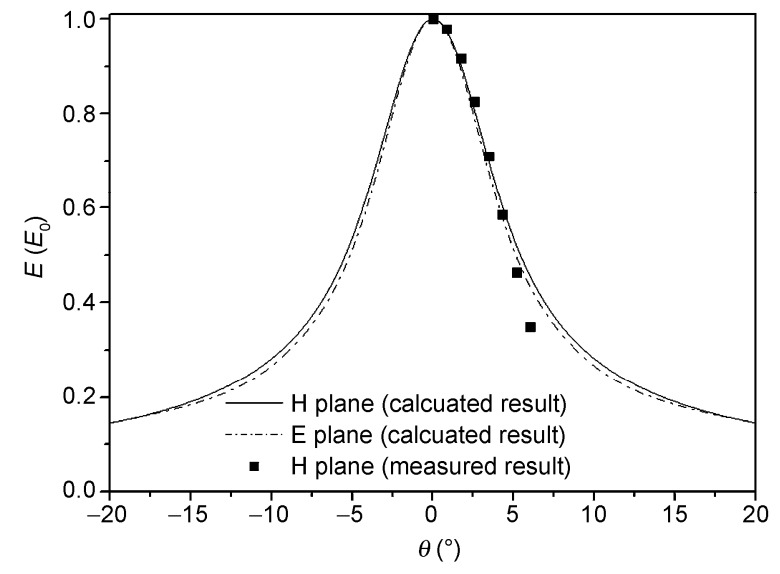

Figure 9 Time domain pattern of a 368 antenna array.

the point-source approximation model (no measurements were taken in the E-plane of the antenna array owing to experimental limitations). Figure 9 shows that the beam width of the electromagnetic pulse from the antenna array is far less than that from a unit antenna in the time domain. The beam width obtained from experiment agrees well with the calculated result within the uncertainty errors, confirming the beam-focusing characteristics of the transient electromagnetic pulses from the array, that in turn gives support to the point-source approximation model as a good model in calculations of electromagnetic pulse parameters of antenna arrays.

\section{Conclusions}

This paper has shown that (1) There exist three regimes, viz. invariance, slow decay, and rapid decay, in the arrayed transient electromagnetic pulse energy propagation. The fundamental physics of these three regimes is the result of energy superpositions in free space rather than the "intrinsic characteristics" independent of continuous electromagnetic waves; (2) the arrayed transient electromagnetic pulses exhibited beam-focusing characteristics; and (3) the pointsource approximation model based on transient electromagnetic pulses demonstrated sufficient precision and practicality in calculations of parameters of electromagnetic pulses radiating from a large antenna array under weak coupling conditions. The parameter calculations presented in this paper were in good agreement with experimental measurements.

This work was supported by the National Natural Science Foundation of China (10804016) and the Electromagnetic Pulse Technology and Investigation (RY0504).

1 Rao S M. Time Domain Electromagnetics. San Diego: Academic Press, 1999

2 Nicolson A M, Bennett C L, Lamensdorf D, et al. Applications of 
time-domain metrology to the automation of broad-band microwave measurements. IEEE Trans Microwave Theory Tech, 1972, 20: 3-9

3 Auston D H. Picosecond optoelectronic switching and gating in silicon. Appl Phys Lett, 1975, 26: 101-103

4 Yang H C, Ruan C L, Zhang K D, et al. Saturation parameters research on linear GaAs photoconductive semiconductor switch. Chinese Sci Bull, 2008, 53: 1516-1522

5 Liu H, Ruan C L. Streamer model in intrinsic GaAs photoconductive semiconductor switch. Chinese Sci Bull, 2008, 53: 2181-2185

6 Wu M H, Zheng X M, Ruan C L, et al. Photoresistances of semiinsulating GaAs photoconductive switch illuminated by $1.064 \mu \mathrm{m}$ laser pulse. J Appl Phys, 2009, 106: 023101

7 Suen J Y, Li W, Taylor Z D, et al. Characterization and modeling of a terahertz photoconductive switch. Appl Phys Lett, 2010, 96: 141103

8 Sun Y Q, Yang $\mathrm{H} \mathrm{C}$, Wu M H, et al. Voltage conversion ratio of photoconductive semiconductor switch in microstrip test circuit. High Power Laser Particle Beams, 2010, 22: 2378-2382

9 Doğan S, Teke A, Huang D, et al. 4H-SiC photoconductive switching devices for use in high-power applications. Appl Phys Lett, 2003, 82: 3107-3109

10 Allen O E, Hill D A, Ondrejka A R. Time-domain antenna characterizations. IEEE Trans Electromagn Compat, 1993, 35: 339-346

11 Shlivinski A, Heyman E, Kastner R. Antenna characterization in the time domain. IEEE Trans Antenna Propagat, 1997, 45: 1140-1149

12 Brittingham J N. Focus wave modes in homogeneous Maxwell's equations: Transverse electric mode. J Appl Phys, 1983, 54: 1179-1189

13 Wu T T. Electromagnetic missiles. J Appl phys, 1985, 57: 2370-2373

14 Wang G, Wang W B, Liang C H. Beam characteristics of short-pulse radiation with electromagnetic missile effect. J Appl Phys, 1998, 83: 5040-5044

15 Shen H M. Electromagnetic missiles from a nonuniform aperture field. Proc SPIE, 1990, 1226: 278-289

16 Louberiel G M, Aurand J F, Dension G J, et al. Optically-activated GaAs switches for ground penetrating radar and firing set applica- tions. In: 12th IEEE International Pulsed Power Conference, 1999 June 27-30, Monterey, CA. 1999. 673-676

17 Islam N E, Schamiloglu E, Fleddermann C B. Characterization of a semi-insulating GaAs photoconductive semiconductor switch for ultra-wideband high power microwave applications. Appl Phys Lett, 1998, 73: 1998-1990

18 Krokel D, Grischkowsky D, Ketchen M B. Sub-picosecond electrical pulse generation using photoconductive switches with long carrier lifetimes. Appl Phys Lett, 1989, 54: 1046-1047

19 David M, Michael D U, Ronald M G, et al. Conductive versus capacitive coupling for cell electroporation with nanosecond pulses. J Appl Phys, 2009, 106: 074701

20 Chalise P R, Perni S, Shama G, et al. Lethality mechanisms in Escherichia coli induced by intense sub-microsecond electrical pulses. Appl Phys Lett, 2006, 89: 153902

21 Yang H C, Ruan C L, Lin W T. Beam scanning and decaying property of linear element planar antenna array. Chin J Radio Sci, 2003, 18: 496-501

22 Yang H C, Ruan C L, Lin W T. The study of axial energy decaying property influenced by pulse waveform. J Electron Infor Tech, 2003, 25: 1430-1435

23 Yang H C, Cui H J, Sun Y Q, et al. High power, longevity gallium arsenide photoconductive semiconductor switches. Chinese Sci Bull, 2010, 55: 1331-1337

24 Sun Y Q, Yang H C. Physical essence of energy transmission characteristics of transient electromagnetic pulse. Chin J Radio Sci, 2010, 25: $323-329$

25 Wu M H, Ruan C L, Yang H C. High-power radiation system with PCSS acting as the photoconductive switch and a scanning-back charging transformer. High Volt Eng, 2007, 33: 143-145

26 Zeng G, Yang X L, Yang H C. Design of full digital time-lapse trigger. Exper Sci Technol, 2008, 6: 3-4

27 Ruan C, Zhao W, Liu B, et al. Laser distribution by optical fiber bundle in the ultra-wideband antenna array. Opt Eng, 2008, 47: 1-6

Open Access This article is distributed under the terms of the Creative Commons Attribution License which permits any use, distribution, and reproduction in any medium, provided the original author(s) and source are credited. 